\title{
Assessment of Pharmacist Dispensing Practices and their Educational Role toward Patients on Oral Cytotoxic Drugs Regimen in Radiation and Isotopes Center at Khartoum, Sudan
}

\author{
Alaa Abdelaziz Mohamed ${ }^{1}$, Mousnad Mohamed Awad ${ }^{2, *}$ \\ ${ }^{1}$ Department of Clinical Pharmacy, University of Khartoum, Khartoum, SUDAN. \\ ${ }^{2}$ Faculty of Pharmacy, International University of Africa (IUA), Khartoum, SUDAN.
}

Received: 03 October 2019;

Accepted: 19 December 2019

*Correspondence to:

Dr. Mohamed Mousnad,

Consultant of Pharmacoeconomics and Pharmacoepidemiology, Assistant Professor, Faculty of Pharmacy, International University of Africa (IUA), Khartoum, SUDAN. Email:mabdalaziz@yahoo.com Copyright: (c) the author(s),publisher and licensee Indian Academy of Pharmacists. This is an open-access article distributed under the terms of the Creative Commons Attribution Non-Commercial License, which permits unrestricted non-commercial use, distribution, and reproduction in any medium, provided the original work is properly cited.

\begin{abstract}
Background: Increasing use of oral cytotoxic chemotherapy among cancer patient in Sudan, rising important concern about safety practicing and using of these drugs. This study aimed to assess and demonstrate pharmacist dispensing pattern and their educational role toward patients on oral cytotoxic drugs regimen in Radiation and Isotopes Center at Khartoum (RICK). Methods: Across-sectional study was carried out at (RICK) hospital pharmacies. Data collected by using self-administered questionnaire distributed to all staff in active works (40 pharmacists) during February 2016. Results: All the participants responded to the questionnaire. The study reported variation among pharmacists when checking prescription. In which $97.4 \%$ of them checked drug name while only $76.9 \%$ calculating the dose according to the body surface area. For the patients-pharmacist counseling and it is effectiveness the study revealed that $77.5 \%$ of pharmacist providing counseling for the patient at the first visit. $75 \%$ of pharmacists counseling the drugs regimen. $28.1 \%$ of them including the drugs side effects and management. The counseling of the drugs handling and storage was registered by $65.6 \%$ of the participants. $53.1 \%$ of the pharmacists advice the patients to swallow the tablets and not crush it. The drugs interaction was counseled by $9.4 \%$ and only $2 \%$ of pharmacists counseled patients about medications teratogenicity. The finding revealed significant association between pharmacist experience and counseling practice by chi-square ( $p$-value $=0.008)$ while, the hospital training and staff attending has on significant association with ( $p$-value=0.922, $p$-value $=0.479$ ). Conclusion: The study concluded that dispensing practice among pharmacists was main divers. Moreover, most of the pharmacists followed no standard when counseling patients and their experience becomes the main source when educating them.
\end{abstract}

Key words: Oral cytotoxic chemotherapy, Safe practicing, Patient education, Side effect management, Pharmacist role, Sudan.

\section{INTRODUCTION}

The administration of oral chemotherapy has been in use since as early as the 1940 s to treat chronic leukemia. ${ }^{[1]}$ After the approving of capecitabine to treat cancer at $1998,{ }^{[2]}$ the development of oral chemotherapy agents has increased dramatically. ${ }^{[1,2]}$ With approving of new targeted molecular therapies, more than $25 \%$ antineoplastic agents currently being developed are oral drugs. ${ }^{[3,4]}$ These shifting oncologists to prescribe oral chemotherapy, as it has many social and economic advantages for patients including increased control and convenience for the patient, potential increase in the quality of life, sustained medication exposure and potential reduction in travel costs and use of health care resources. ${ }^{[1]}$ While there are several advantages to prescribing oral chemotherapy, one must bear in mind that home-based chemotherapy may continue for some time without professional supervision. The intermittent nature of treatment regimens may be confusing to some patients and their families and non-compliance through misinterpretation carries the risk of serious harm..$^{[5-7]}$

Oral cytotoxic chemotherapy (OCCT) has the same risk as parenteral chemotherapy in terms of toxicities and potential for harm from medication, due to the narrow therapeutics index of these drugs. ${ }^{[5,6,8]}$ Although there are few publications comparing chemotherapy errors that occur with oral versus intravenous administration, ${ }^{[9]}$ there are several concerns that arise, including patient adherence, management of adverse reactions, drugs interaction, storage and handling. Which creates challenges and increase responsibilities for healthcare professionals in patient education. ${ }^{[5,6,10]}$

Safe practicing of oral cytotoxic drugs involves the processes of prescribing, dispensing, patient education, administration, handling and storage of these drugs. ${ }^{[1]]}$ This study concentrates on the implementation of safety practices guidelines of these drugs among pharmacies staff. No recent study was done in Sudan that indicates safety of using these oral cytotoxic chemotherapies. The main objective of this study is to demonstrate safeguard dispensing guideline application and assessing pharmacist educational role toward patients on oral cytotoxic chemotherapy. The study was focused into four categorical points: drug regimen, side effect and management, handling and storage and drug/food/herbs interaction. 


\section{Mohamed and Awad.: Assessment of Oral Cytotoxic Drugs, Sudan}

\section{METHODS}

This study is Qualitative, descriptive, cross-sectional study. It was carried out at the pharmacies of Radiation and Isotopes Center of Khartoum. Targeted all pharmacists on active work during February 2016.

\section{Data collection method}

Data were collected by using questionnaire. It was focused on the dispensing of five oral cytotoxic chemotherapies (methotrexate, thalidomide, 6-mercaptoburine, melphalan and hydroxyurea) which were dispensed at the hospital. The structure of it was based on the similar literature and the data from the Drug Information Center (DIC) at RICK hospital. A pilot study was done on five pharmacists to validate and adjust it.

\section{Sample selection}

The study covers all pharmacists on active work load during February. The total number of pharmacists was 40 .

\section{Study protocol}

Participants filled the questionnaire after clarify the aim of the study to them.

\section{Data management and statistical analysis}

Data were tabulated using excel 2010 and analyzed using statistical package analysis of social science (SPSS v.20). Binary logistic regression analyses were carried out for the dependent variable 'counseling to first visit patients'.

\section{Ethical considerations}

Approval from the hospital authorities and consultants running the pharmacies was obtained. Also, verbal informed consent was obtained from pharmacists prior to receiving questionnaire.

\section{RESULTS}

\subsection{Demographic data of RICK pharmacies and their} staff:

At the RICK hospital, there are seven pharmacies dispensing chemotherapy, four of which were specified to dispense oral cytotoxic drugs. Pharmacies staff are shifted constantly through all pharmacies, so all of them dispensed oral cytotoxic drugs. The range of daily prescriptions seen by all medical staff including oral and IV was around 640-810 as shown in Table 1 while, Figure 1 represent prescription seen per candidate.

Experience varies among participants with $37.5 \%$ having $1-3$ years of experience, $27.5 \%$ having $4-10$ years, $20 \%$ with less than one year and $15 \%$ with more than 10 years of experience.

Regarding staff training on oral chemotherapy, $73 \%$ said they received training and $47.5 \%$ regularly attend CPD at the hospital.

Figure 2 represent chi-square $(p$-value $=0.008)$ test, which revealed significant association between experience and counseling practice.

For training and regularity of attending it, chi-square test with ( $p$-value $=0.922$, $p$-value $=0.479)$ revealed no significant association of training and attending it with the candidate willingness to counsel patient arriving for the first time as shown in Figures 3,4.

\subsection{Dispensing:}

At RICK hospital there are six types of oral cytotoxic drugs, the frequencies of dispensing of these drugs by candidates was shown to have a close percentage; chlorambucil registered the highest one, with $56.8 \%$, followed by methotrexate and 6-mercaptoburine with $54.1 \%$, hydroxyurea and thalidomide with $35.1 \%$, finally melphalan with $29.7 \%$.

Regarding the pharmacist concern when checking prescriptions, most of the staff registered a high percent of checking dates and drug names $(94.9 \%$ and $97.4 \%$, respectively). The time interval of the patient visits and if the prescription written by consultant or registrar, this was checked by $84.6 \%$ of staff. The other information registered less consideration such as dosage calculation according to body surface area, appropriateness of drugs with diagnosis and lab investigations by $76.9 \%, 59 \%$ and $56.4 \%$ respectively.

\subsection{Effectiveness of pharmacist-patient consultations:}

$77.5 \%$ of pharmacist providing counseling for the patient at the first visit.

Figure 5 represent the variation among pharmacists when they are counseling patients. These were divided into fifth categories.

\subsubsection{Firstly, treatment duration and regimen:}

$96.9 \%$ of pharmacist counsel treatment duration while, drug regimen has less priority in the counseling by $75 \%$ of pharmacists.

\subsubsection{Secondly, Side effects and managements:}

Regarding the side effects $28.1 \%$ of pharmacists counseled patients, while $85 \%$ of pharmacists received complaints about drugs. Nausea is the most reported complaint by pharmacists by $84.8 \%$. The management of these complaints represented in Table 2 the result showed that $62.5 \%$ of the pharmacy staff referred patients immediately to their doctors while the Kruskal-Wallis test (Figure 6) revealed significant variation in the response actions made by the pharmacist with experience $(\mathrm{F}=8.996$, $p$-value $=0.029)$. In which the more experienced pharmacists clarify side effects and complaints of these drugs before referring patient.

\subsubsection{Thirdly, Handling and storage:}

$65.6 \%$ of pharmacists said they are counseled the patient about handling and storage. In fact there are many aspects to ensure patient knowledge about the proper handling and storage, the most essential point is to avoid direct contact of these drugs with skin and avoiding tablet or capsule crushing. $53.1 \%$ of pharmacists counseled that point with patients.

\subsubsection{Fourthly, drugs/ herbs/ interaction:}

The serious drugs herb interaction reported as the lowest present $(9.4 \%)$ of the pharmacist consultation priority.

\subsubsection{Fifthly, Essential advice and precaution:}

Regarding the general advice to the patients (Figure 7), 76\% of pharmacists advised patients to take their drugs before or after meals to avoid interactions, while only $2 \%$ of pharmacists counseled patients about teratogenicity of these drugs and advised them to avoid pregnancy. 


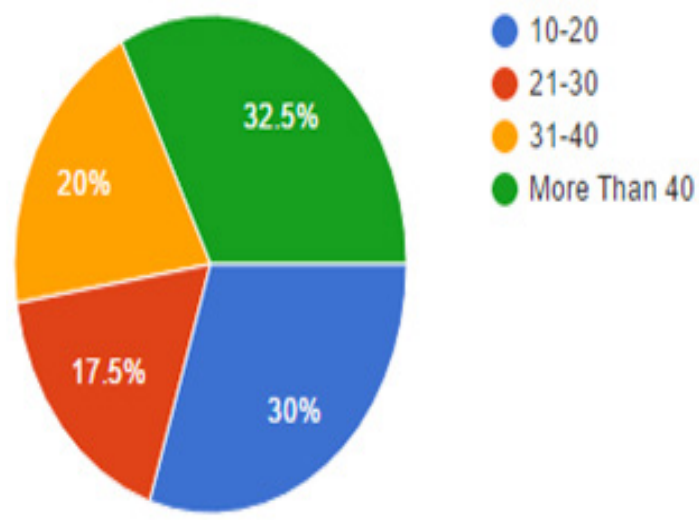

Figure 1: Number of prescriptions dispensed per day by candidate.

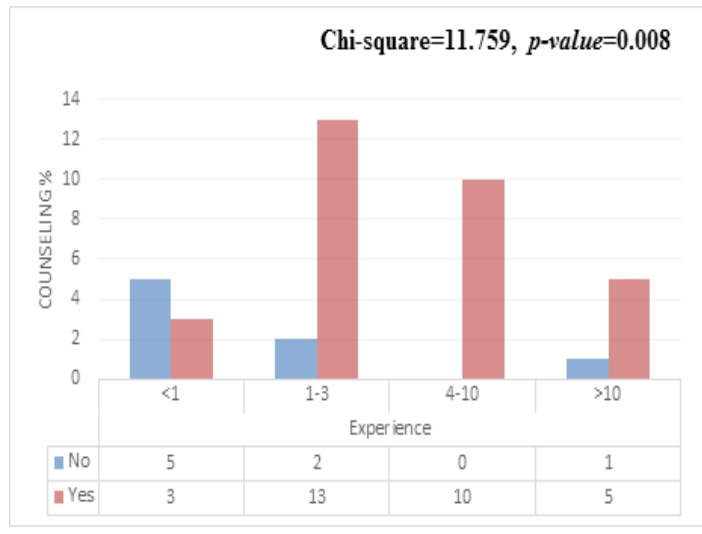

Figure 2: Cross-tabulation of counseling versus experience groups.

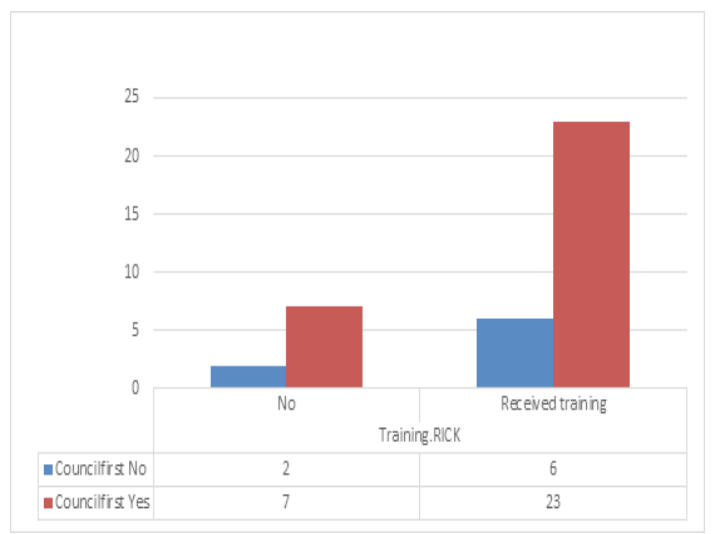

Figure 3: Cross-tabulation of counseling versus training.

\subsection{The pharmacists' parameter correlations:}

Table 3 represents the logistic regression model assessing counseling parameters for the first visit patients between the participants.

\section{DISCUSSION}

This study is one of fewer studies in Sudan concerned to assess pharmacist role toward cancer patients in practicing and using of oral cytotoxic drugs in a safely manner.

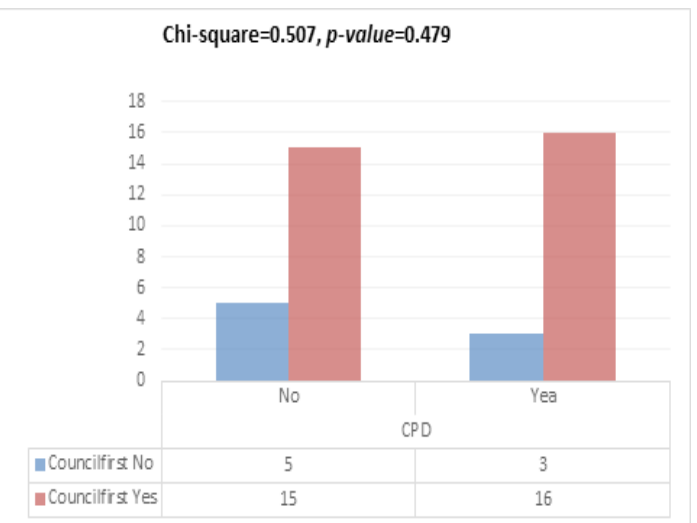

Figure 4: Cross-tabulation of counseling versus regularity of attending training.

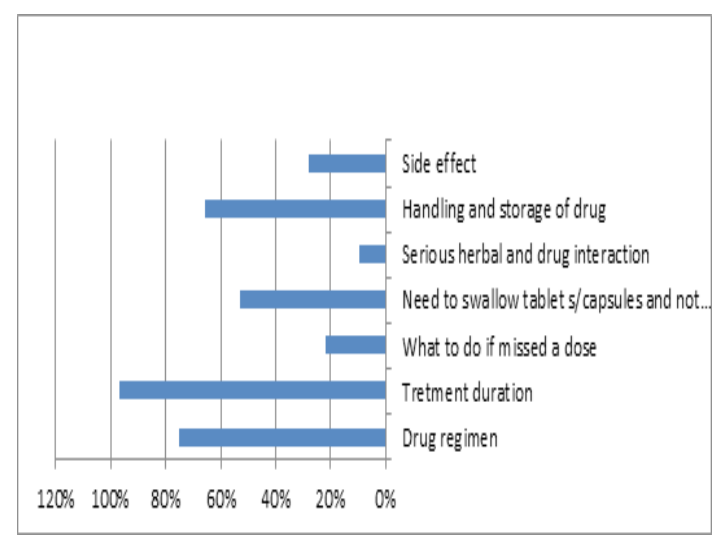

Figure 5: Counseling points implemented by pharmacist.

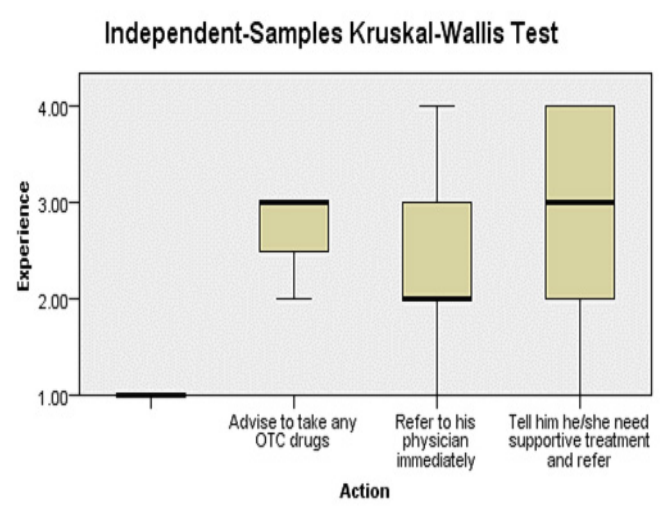

Figure 6: Mean ranks for the Kruskal-Wallis test.

The study showed a high participation rate as it covers all pharmacies staff members in active work load excluding participants of the pilot study to standardize the questionnaire. The drugs are dispensing in four pharmacies (central, public, pediatric and oral pharmacies). Staff members shifted along pharmacies and they dispense oral cytotoxic chemotherapy. Whereas no fixed data which calculate the percentage of prescribed chemotherapy, the rate of oral chemotherapy prescriptions was shown to be high as the pharmacists reported high percent of dispensing. The dispensing of IV prescriptions and oral medication at the same pharmacies may lead to under focusing to patients whom taken the oral medications. As stated in Canadian guideline ${ }^{[12]}$ 


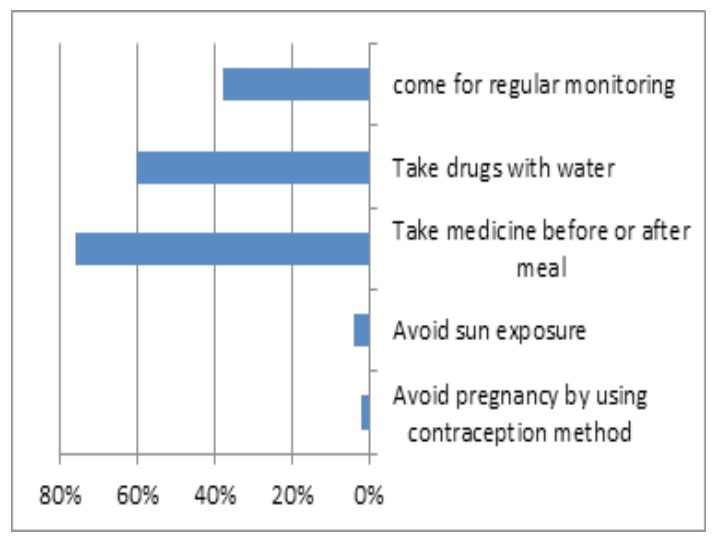

Figure 7: Percentage of pharmacists giving general advice to the patients.

the intravenous therapy take the opportunities while, the oral chemotherapy lack of standardized process to safely self-administered. Indeed these homesitting cytotoxic therapies have the same risk of the parental.

On the other hand, the variation of demographic data among the participants only the experience revealed significant association with counseling practice. As candidates with experience of less than year were less likely to attempt counseling of patients receiving treatment for the first-time; as experience went up, the counseling became the rend.

In addition, the candidates training and the regularity of attending it, have no significant association with patient counseling whom are arriving for the first time. These may be due to generalization of these training on all the chemotherapy (as acknowledge by DIC) and did not specified OCCT with supporting of how to improve the patient education. By revising the literature, continuous education and training for pharmacy staff has an important setting in the guideline generated for oral chemotherapy. ${ }^{[5,12]}$ As stated in recommendations from ASCO at 2013:[13] healthcare professionals should attend orientation programs and routine training courses specific to their roles. The training programs should be approved by an oncology organization or appropriate local organizations.

Parallel with this study, the previous published studies show variable percentage for applying continuous training program. For example, in Bourmaud et al. (2014), ${ }^{[3]} 36 \%$ of the clinicians provided training to the other healthcare team members whom will educate their patients.

Study had done in America ${ }^{[14]}$ reported that, one third of organizations provided special training for those who responsible for patient education. Moreover many studies from the literature identify gaps in the pharmacists knowledge regarding OCCT and their confidence to educate patients. For example, in study across Canada ${ }^{[15]}$ indicate less than $10 \%$ of community pharmacists felt confident in educating patients and $24 \%$ of them have knowledge about common dose calculation. Another study in Saudi Arabia ${ }^{[16]}$ represent that pharmacist knowledge were suboptimal to educate patients and it reported that $20 \%$ have adequate knowledge and only quarter are confident in educating cancer patients. Those studies and our study are highlighting to the diversity in applying continuous pharmacists training program for OCCT.

The drug information center of the hospital standardized prescription information which are matched many standard and guidelines. ${ }^{[5,10,13]}$ The prescriptions in RICK hospital are hand written and it was attached with patient file when coming at the pharmacy for dispensing. May studies ${ }^{[3,4,17]}$
Table 1: Pharmacies categorization and total number of prescriptions (source drug information center (DIC) at RICK hospital).

\begin{tabular}{|l|l|l|}
\hline $\begin{array}{l}\text { Pharmacies at } \\
\text { RICK }\end{array}$ & $\begin{array}{l}\text { Total number of } \\
\text { medical staff at } \\
\text { pharmacy }\end{array}$ & $\begin{array}{l}\text { Average number } \\
\text { prescriptions/day }\end{array}$ \\
\hline Public pharmacy & 3 & $90-100$ \\
\hline Central pharmacy & 17 & $150-200$ \\
\hline Day care pharmacy & 5 & $80-100$ \\
\hline Clinical pharmacy & 9 & $80-100$ \\
\hline Oral pharmacy & 3 & $120-150$ \\
\hline Drugs information office & 3 & $80-100$ \\
\hline Pediatric pharmacy & 5 & $40-60$ \\
\hline
\end{tabular}

Table 2: Pharmacist's actions in response to reported complaints.

\begin{tabular}{|l|l|l|}
\hline & Frequency & Percent \\
\hline Refer to his physician immediately & 25 & 62.5 \\
\hline Tell him he/she need supportive treatment and refer & 9 & 22.5 \\
\hline Missing response & 3 & 7.5 \\
\hline Advise to take any OTC drugs & 3 & 7.5 \\
\hline Total & 40 & 100.0 \\
\hline
\end{tabular}

\section{Table 3: Logistic regression model assessing determinants of counseling to first visit patients between the pharmacist participants.}

\begin{tabular}{|l|l|l|l|l|}
\hline & B & Wald & Sig. & $\begin{array}{l}\text { Odd } \\
\text { ratio }\end{array}$ \\
\hline Age 20-30 Years & -1.539 & 1.055 & 0.304 & 0.214 \\
\hline Age 31-50 Years & -1.539 & 1.055 & 0.304 & 4.662 \\
\hline Experience <1 Years & -5.024 & 5.378 & 0.020 & 0.007 \\
\hline Experience 1-3 Years & -2.039 & 1.424 & 0.233 & 0.13 \\
\hline Prescriptions dispensed 10-20 & 2.084 & 1.684 & 0.194 & 8.033 \\
\hline Prescriptions dispensed 21-30 & 2.447 & 2.133 & 0.144 & 11.556 \\
\hline Prescriptions dispensed 31-40 & 0.664 & 0.224 & 0.636 & 1.942 \\
\hline Constant & 4.695 & 2.637 & 0.104 & 109.354 \\
\hline
\end{tabular}

reported that the using of the hand written prescription was commonly prevalent, while the using of electronic system prescription was highly encouraged and stated in many guidelines ${ }^{[11,12]}$ which may reduce errors.

The pharmacists reported diversity when checking prescription. They are considered the dates and drug name as crucial points for them to Check. Dosage according to body surface area, appropriateness of drugs and lab investigations are in less priority with variable percentage among pharmacists whom check it. These result was resemble to the O'Bryant ${ }^{[14]}$ and Bourmaud $e t$ al. ${ }^{[3]}$ studies in which lack of standardization was reflected in the prescriptions information.

$77.5 \%$ of pharmacists participated in educating patients for the first visit only verbally without using special written educational materials. When 


\section{Mohamed and Awad.: Assessment of Oral Cytotoxic Drugs, Sudan}

comparing these results with the standard guidelines ${ }^{[5,13]}$ it was stated that the patients should be educated using verbal and written manifestation. In addition, the awareness should be increased toward the needs of regular monitoring arrangements. The result of our study was shown that $38 \%$ of pharmacists told patients to come for regular monitoring and $21.9 \%$ of them counseling the missing dose issue. Moreover, only $2 \%$ of pharmacists counseled patients about teratogenicity of these drugs and advised them to avoid pregnancy. Moreover many studies emphasize the role pharmacist specifically in the first consultation visit for example, in Simchowitz et al. ${ }^{[18]}$ (2010), pharmacist is considered as an important information source after clinician, but the participants believed that the initial prescribing encounter should have included more education. While in Bourmaud et al. ${ }^{[3]}$ study $28 \%$ of the centers had developed addition of therapeutics education materials for the patient at the first visit, while $12 \%$ of the clinicians who participated in this study holding regular workshops with their patients. In CondeEstévez et al..$^{[4]} 44.2 \%$ of hospitals classified as level II and performed an initial counseling visit with a pharmacist by $98.8 \%$. It also providing written educational materials and monitoring adherence. In the study published in the BMJ in $2007,{ }^{[17]}$ they found that $95 \%$ of the patient education was by the pharmacist, while the physician shared responsibility for educating patient.

Although $85 \%$ of pharmacists received complaints about drugs, only $28.1 \%$ of them counseled patients about the side effects of the drugs. The more experienced pharmacists have significant role in response to complaints and clarifying side effects. In previously published studies such as Bourmaud et al. (2014), ${ }^{[3]} 39 \%$ of the clinicians they said that they recalled at least one serious adverse event over one year and $80 \%$ of them said that they are worried about the risks of oral chemotherapy. In Simchowitz et al. ${ }^{[18]}$ the participants raised concerns regarding their lack of preparedness for side effects and their unfamiliarity with the possible techniques to mitigate drug toxicity.

Regarding drugs interaction the study revealed $9.4 \%$ of the pharmacists discuss the possible interactions of drugs regimen with herbs or other medicine. $76 \%$ of them advised patients to take their drugs before or after meals to avoid interactions. In Segal et al..$^{[19]}$ noted that drug- drug or drugfood interactions should be considered when using oral chemotherapeutic. Interaction of these drugs could be with over the counter (OTC) medication (e.g. methotrexat (MTX) with Nonsteroidal Anti-infammatory drugs NSAID or aspirin). ASCO guideline ${ }^{[13]}$ and Carrington $\mathrm{C}^{[10]}$ stated that reviewing the current medications should be performed with the patient to identify potential medication interactions or interference with dietary intake.

For the proper drug handling and storage at home setting, more than half of pharmacists direct patients how to deal with their medicine. Clinical guidelines ${ }^{[5,11]}$ recommended using of label for each medication. In another studies ${ }^{[18,20]}$ participants were worried about proper handling by non-patients and hand washing before and after drugs administration.

The second logistic model studied the correlation between three variables on pharmacists (ages, years of experiences and work load) counseling patients at first visit. The model reveled significant variable interaction, goodness-of-fit chi-square $=13.226, p$-value $=0.004$ with the predictive ability of $28.8 \%$ of the variance in counseling stance. Moreover, neither age nor dispensing load were nominated as significant contributors; however, having experience less than one year reduced the chance of initiating counseling by $93 \%$ as compared to more experienced candidates (Wald $=5.378, p$-value $=0.020$ and $\mathrm{OR}=0.007$ ). Pharmacist's age showed increased odds of prevalence with the 20-30 years aged candidates as they were 4.662 times more liable to initiate counseling compared to $31-50$ years aged pharmacists who tended to have $79.6 \%$ lesser probability of counseling initiation.

\section{CONCLUSION}

In conclusion, the implementation of safeguard dispensing guideline of oral cytotoxic chemotherapy in RICK hospital was shown to be diverted among participants. In addition, the educational role of pharmacist toward these drugs was based mainly on their experiences. Improvement of pharmacy staff training program with specification of oral chemotherapy was highly recommended. The results suggest that, using of check-list points in patients counseling will make it uniformly, reduce medication error and achieving the therapeutic goals.

\section{ACKNOWLEDGEMENT}

This paper was part of a thesis at the University of Khartoum, Sudan submitted by Alaa Mohamed and supervised by Assistant Prof. Mohammed Mousnad. The authors would like to thank faculty members, staff at faculty of Pharmacy University of Khartoum for their precious suggestions. Many thanks also goes to RICK hospital and pharmacies staff for their cooperation.

\section{CONFLICT OF INTEREST}

The authors declare that they do not have conflict of interest to disclose.

\section{ABBREVIATIONS}

OCCT: Oral cytotoxic chemotherapy; DIC: Drug Information Center; RICK: Radiation and Isotopes Center of Khartoum; IV: Intravenous; CPD: Continuous Professional Development; ASCO: American Society of Clinical Oncology; BMJ: British Medical Journal; OTC: Over the counter; MTX: methotrexat; NSAID: Nonsteroidal Anti-infammatory drugs.

\section{REFERENCES}

1. Aisner J. Overview of the changing paradigm in cancer treatment: Oral chemotherapy. American Journal of Health-System Pharmacy. 2007;64(9).

2. Karen L, Milkiewicz PHD. Research Scientist II, Teva Pharmaceuticals, Oral Chemotherapy and the Pharmacist's Role: Providing Pharmaceutical Care for Cancer Patient, Pharm Con, Inc. has been approved by the American Nurses Credentialing Center's Commission on Accreditation. 2015.

3. Bourmaud A, Pacaut C, Melis A, Tinquaut F, Magné N, Merrouche Y, Chauvin F. Is oral chemotherapy prescription safe for patients? A cross-sectional survey. Annals of Oncology. 2014;25(2):553.

4. Conde-Estévez D, Salas E, Albanell J. Survey of oral chemotherapy safety and adherence practices of hospitals in Spain. International Journal of Clinical Pharmacy. 2013;35(6):1236-44

5. Grampians Regional Palliative Care Team, Clinical Guidelines for the Administration of Oral Chemotherapy Agents in the Community Setting For Health Care Professionals, Victoria. 2013.

6. SHPA Committee of Specialty Practice in Cancer Services, Standards of Practice for the Provision of Oral Chemotherapy for the Treatment of Cancer. Journal of Pharmacy Practice and Research. 2007;37(2).

7. Schulmeister L. Chemotherapy medication errors: Descriptions, severity and contributing factors. Oncol Nurs Forum. 1999;26(6):1033-42.

8. Goodin S, Griffith N, Chen B, Chuk K, Daouphars M, Doreau C, et al. Safe handling of oral chemotherapeutic agents in clinical practice: Recommendations from an international pharmacy panel. Journal of Oncology Practice. 2011;7(1):7-12.

9. Blecher C, Barefoot J, Davis D, et al. A team approach toward promoting patient adherence to oral chemotherapy protocols. Oncol Nurs Forum. 2008;35:537. (abstr 2985).

10. Carrington C. Safe use of oral cytotoxic medicines. Australian Prescriber. 2013;36(1):9-12.

11. Royal Cornwall Hospitals NHS trust. The Safe Handling and Administration of Cytotoxic Products for the Treatment of Cancer. 2015.

12. Canadian Association of Provincial Cancer Agencies. Oral Cancer Drug Therapy Safe Use and Safe Handling Guidelines. 2015.

13. Neuss MN, Polovich M, McNiff K, Esper P, Gilmore TR, LeFebvre KB, et al. 2013 updated American Society of Clinical Oncology/Oncology Nursing Society chemotherapy administration safety standards including standards for the safe administration and management of oral chemotherapy. Journal of Oncology 


\section{Mohamed and Awad.: Assessment of Oral Cytotoxic Drugs, Sudan}

Practice. 2013;9(2S):5s-13s.

14. O'Bryant CL. Community pharmacists' knowledge of and attitudes toward oral chemotherapy. Journal of the American Pharmacists Association. 2008;48(5):632 9.

15. Abbott R, Edwards S, Whelan M, Edwards J, Dranitsaris G. Are community pharmacists equipped to ensure the safe use of oral anticancer therapy in the community setting? Results of a cross-country survey of community pharmacists in Canada. Journal of Oncology Pharmacy Practice. 2014;20(1):29-39.

16. Mekdad SS, AISayed AD. Towards safety of oral anti-cancer agents, the need to educate our pharmacists. Saudi Pharmaceutical Journal. 2017;25(1):136-40.

17. Weingart SN, Flug J, Brouillard D, Morway L, Partridge A, Bartel S, et al. Oral chemotherapy safety practices at US cancer centers: Questionnaire survey. BMJ. 2007;334(7590):407.

18. Simchowitz B, Brouillard D, Maureen C, Weingart SN. Perceptions and experiences of patients receiving oral chemotherapy. Clinical Journal of Oncology Nursing. 2010;14(4):447.

19. Segal EM, Flood MR, Mancini RS, Whiteman RT, Friedt GA, Kramer AR, et al. Oral chemotherapy food and drug interactions: A comprehensive review of the literature. Journal of Oncology Practice. 2014;10(4):e255-68.

20. Chan A, Leow YC, Sim MH. Patients' perspectives and safe handling of oral anticancer drugs at an Asian cancer center. Journal of Oncology Pharmacy Practice. 2009;15(3):161-5.

Cite this article as: Mohamed AA, Awad MM. Assessment of Pharmacist Dispensing Practices and their Educational Role toward Patients on Oral Cytotoxic Drugs Regimen in Radiation and Isotopes Center at Khartoum, Sudan J Pharm Pract Community Med. 2019;5(4):63-8. 